\title{
sciendo
}

Int. J. of Applied Mechanics and Engineering, 2021, vol.26, No.3, pp.84-103

DOI: 10.2478/ijame-2021-0037

\section{WAVELET-BASED NUMERICAL SOLUTION FOR MHD BOUNDARY-LAYER FLOW DUE TO STRETCHING SHEET}

\author{
Harinakshi KARKERA and Nagaraj N. KATAGI* \\ Manipal Institute of Technology, Manipal Academy of Higher Education, Manipal, 576104, INDIA \\ E-mail:nn.katagi@manipal.edu
}

\begin{abstract}
In this paper, a two-dimensional steady flow of a viscous fluid due to a stretching sheet in the presence of a magnetic field is considered. We proposed two new numerical schemes based on the Haar wavelet coupled with a collocation approach and quasi-linearization process for solving the Falkner-Skan equation representing the governing problem. The important derived quantities representing the fluid velocity and wall shear stress for various values of flow parameters $M$ and $\beta$ are calculated. The proposed methods enable us to obtain the solutions even for negative $\beta$, nonlinear stretching parameter, and smaller values of the magnetic parameter $(M<1)$ which was missing in the earlier findings. Numerical and graphical results obtained show an excellent agreement with the available findings and demonstrate the efficiency and accuracy of the developed schemes. Another significant advantage of the present method is that it does not depends on small parameters and initial presumptions unlike in traditional semi-analytical and numerical methods.
\end{abstract}

Key words: MHD flow, boundary layer, quasi-linearization, Haar wavelets.

\section{Introduction}

The knowledge of boundary-layer flow phenomena is an important prerequisite to many physical problems in manufacturing industries that include stretching of polymer and metal sheets. This is also experienced in several other practical applications such as the cooling and extrusion of plastic or metal sheets, paper production, glass and polymer industries, fibre processing, etc. More precisely, it is important that one must know the rate of cooling and the boundary-layer control of the stretching material in a moving or quiescent fluid to achieve the desired outcomes in such situations. Thus, a variable magnetic field is needful to regulate an electrically conducting cooling fluid and to control the cooling system. Evidently, the study of a magnetohydrodynamic (MHD) boundary-layer flow of a viscous fluid due to a stretching sheet is found to be very necessary and has received considerable interest among researchers in recent days. In fact, the effect of an MHD boundary-layer flow of a nanofluid associated with heat transfer, porous stretching medium, etc., has been addressed extensively in the literature due to its important impacts on the behaviours of the fluid flow [1-3].

In practice, the governing physical problem is converted into an equivalent mathematical one, which is a third-order nonlinear ordinary differential equation known as the Falkner-Skan equation. This equation is the most celebrated equation and provides a considerable contribution to the development of boundarylayer theory in fluid mechanics. Crane [4] presented a brief report on the two-dimensional boundary-layer flow caused by linear stretching and obtained the analytical solution. Followed by this, many researchers [5-7] studied the salient features of hydromagnetic flow and heat transfer over a stretching sheet. They obtained the closed-form solutions and eigen solutions at specific values of flow parameters. Chiam [8] analysed the problem of boundary-layer flow of a viscous fluid due to a stretching sheet with a power-law velocity distribution in the presence of a magnetic field. A brief literature survey on the flow past a stretching sheet can be found in Liao [9]. He obtained a dual solution for impermeable stretched surfaces using an

\footnotetext{
${ }^{*}$ To whom correspondence should be addressed
} 
analytic technique, namely the homotopy analysis method. Recently, Kudenatti [10] investigated an MHD boundary-layer flow over a stretching boundary and found an analytical solution in terms of error and exponential function. Several researchers, namely Hayat et al. [11], Rashidi [12], Mehmood et al. [13] analysed the problem considered by [8] using semi-analytical methods such as the Adomian decomposition method, differential transform method, and homotopy analysis method, respectively. Due to the limitations of the domain of radius of convergence, semi-analytical methods diverge when the boundary-layer variable goes to infinity. To overcome this difficulty to a certain extent they used the above semi-analytical methods coupled with Padé approximants. However, these methods fail to converge the solution for quite smaller values of $M$ for specific negative $\beta$ values (where $M$ and $\beta$ are respectively the magnetic parameter and the nonlinear stretching parameter which will be defined later). Therefore, the problem demands innovative new numerical methods for their analysis. Motivated by this fact, the wavelet methods have received a special attention of researchers.

Wavelet methods are recognized as one of the most powerful and rapidly evolving methods for obtaining a numerical solution of ordinary and partial differential equations with growing applications in science and engineering. The advancement of the Haar wavelet method in the subject of numerical analysis started in 1997 when Chen and Hsiao [14] developed an operational matrix of integration to solve differential equation models of dynamic systems. They introduced the concept of expanding the function corresponding to highest derivative of differential equation into Haar series. Since then the solutions of differential/integral equations in a wavelet framework have grown. Following the pioneering work of Chen and Hsiao [14], Lepik [15-18] further modified and generalized the Haar wavelet method with a uniform grid for the solution of differential, integral, integro-differential and fractional integral equations. Consequently, several other researchers have been contributing substantially in the study of Haar wavelets [19-22]. In recent analyses, the Haar wavelet method in conjunction with quasilinearization approach has become quite popular in solving the nonlinear boundary value problems. Saeed and Rehman [23], Kaur et al. [24], Jiwari [25], successfully applied the Haar wavelet along with the quasilinearization technique to confront the nonlinear aspects of physical problems. However, in most of the above works, the researchers considered only the problem with finite domain. As a vital part of this work, we attempt to extend this method to examine the results for higher order nonlinear boundary value problems with semi-infinite or infinite domain and thus making the method more useful in real-world applications.

In the present study, a novel numerical method based on the Haar wavelet has been developed and successfully applied to a strongly nonlinear boundary value problem with semi-infinite domain representing the two-dimensional MHD steady flow of a viscous fluid due to a stretching sheet. Towards this goal, we proposed two numerical schemes based on Haar wavelet approximations: the Haar wavelet collocation method (HWCM) and Haar wavelet quasilinearization method (HWQLM). These methods are notable for the following benefits:

- The method provides a simple solution procedure due to inherent features such as orthonormalization, compact support, simple explicit expression, and the hierarchy of solution approximation.

- The simplicity, sparsity of Haar wavelet matrices, and also the solution representation with significantly a smaller number of wavelet coefficients increase the speed of convergence of the method.

- Unlike semi-analytical methods, the proposed method avoids the restriction of existence of small perturbation parameters and also does not require the initial assumption of base function.

- It does not demand the initial guesses for the solution at each and every case corresponding to values of parameters under study, unlike in traditional numerical methods.

- The method is very convenient for solving boundary value problems since the boundary conditions are taken care of automatically and also, it is very effective for treating singularities (since they can be interpreted as intermediate boundary conditions).

- The method works well even for problems with semi-infinite or infinite domains.

- It promotes the possibility of the implementation of fast algorithms with minimal calculations.

This paper is structured as follows: Section 2 is devoted to a brief review of Haar wavelets. The mathematical formulation of the problem is described in Section 3. In Section 4, we develop the solution 
procedure based on HWCM and HWQLM. Section 5 is mainly concerned with the discussion of the obtained results followed by concluding remarks in Section 6.

\section{Haar wavelets}

Haar wavelets have received most attention among wavelet families due to their beneficial features such as orthonormalization, symmetric, compact support and simple explicit expression for scaling and wavelet functions. They were first introduced by Alfred Haar in 1910, as pairs of piecewise constant functions. The Haar basis provides a very efficient representation of functions that consist of smooth, slowly varying segments punctuated by sharp peaks and discontinuities. The Haar wavelet family for $x \in[0,1)$ is defined as [26]

$$
h_{i}(x)= \begin{cases}1, & x \in\left[\xi_{1}(i), \xi_{2}(i)\right), \\ -1, & x \in\left[\xi_{2}(i), \xi_{3}(i)\right), \\ 0, & \text { elsewhere }\end{cases}
$$

where

$$
\xi_{1}(i)=\frac{k}{m}, \quad \xi_{2}(i)=\frac{k+0.5}{m}, \quad \xi_{3}(i)=\frac{k+1}{m}
$$

and $j=0,1, \ldots, J$ stand for the dilation parameter, $J$ is the resolution level. The translation parameter $k=0,1, \ldots, m-1$ indicates the location of the particular square wave $\left(m=2^{j}\right)$ and $i=m+k+1$ is the wavelet number. The minimum value of $i$ is 2 (i.e., $m=1, k=0$ ) and the maximum value of $i$ is $2^{J+1}$. For $i=1$, the function $h_{l}(x)$ is the scaling function for the family of Haar wavelets defined as

$$
h_{l}(x)= \begin{cases}1, & x \in[0,1), \\ 0, & \text { elsewhere }\end{cases}
$$

The integration of the Haar functions $h_{i}(x)$ can be evaluated as

$$
p_{1, i}(x)=\int_{0}^{x} h_{i}(t) d t= \begin{cases}x-\xi_{1}(i), & x \in\left[\xi_{1}(i), \xi_{2}(i)\right), \\ \xi_{3}(i)-x, & \left.x \in\left[\xi_{2}(i), \xi_{3}(i)\right)\right) \\ 0, & \text { elsewhere. }\end{cases}
$$




$$
\begin{aligned}
& p_{2, i}(x)=\int_{0}^{x} p_{1, i}(t) d t= \begin{cases}\frac{1}{2}\left(x-\xi_{1}(i)\right)^{2}, & x \in\left[\xi_{1}(i), \xi_{2}(i)\right), \\
\frac{1}{4 m^{2}}-\frac{1}{2}\left(\xi_{3}(i)-x\right)^{2}, & x \in\left[\xi_{2}(i), \xi_{3}(i)\right), \\
\frac{1}{4 m^{2}}, & x \in\left[\xi_{3}(i), 1\right), \\
0, & \text { elsewhere. }\end{cases} \\
& p_{3, i}(x)=\int_{0}^{x} p_{2, i}(t) d t= \begin{cases}\frac{1}{6}\left(x-\xi_{1}(i)\right)^{3}, & x \in\left[\xi_{1}(i), \xi_{2}(i)\right), \\
\frac{1}{4 m^{2}}\left(x-\xi_{2}(i)\right)+\frac{1}{6}\left(\xi_{3}(i)-x\right)^{3}, & x \in\left[\xi_{2}(i), \xi_{3}(i)\right), \\
\frac{1}{4 m^{2}}\left(x-\xi_{2}(i)\right), & x \in\left[\xi_{3}(i), 1\right), \\
0, & \text { elsewhere. }\end{cases}
\end{aligned}
$$

Similarly

$$
p_{\alpha+1, i}(x)=\int_{0}^{x} p_{\alpha, i}(t) d t, \quad \alpha=3,4, \ldots
$$

For convenience of computations, the matrix form is introduced: $H(i, l)=h_{i}\left(x_{l}\right), P \alpha(i, l)=p_{\alpha, i}\left(x_{l}\right)$, $\alpha=1,2, \ldots$, which are of order $2^{J+1} \times 2^{J+1}$. The sparsity pattern of the Haar matrix $H$ and its first integral $P 1$ are shown in Fig.1. for $J=5$ and $J=6$, respectively. We notice that as the size of the matrices increases (i.e., $J$ increases), the sparsity of the matrices also increases. This 'non-dense' property of Haar matrices and inverse matrices makes the Haar wavelet transform faster when compared with other transforms and also more convenient for computer implementations. Any function $y(x) \in L^{2}[0,1)$ can be expanded in a Haar series with an infinite number of terms as

$$
y(x)=\sum_{i=1}^{\infty} a_{i} h_{i}(x)
$$

where $a_{i}=2^{j} \int_{0}^{1} y(x) h_{i}(x) d x, j \geq 0, i=1,2, \ldots$, are the Haar coefficients. The above series terminates at finite terms if $y(x)$ is a piecewise constant or may be approximated as piecewise constants. 

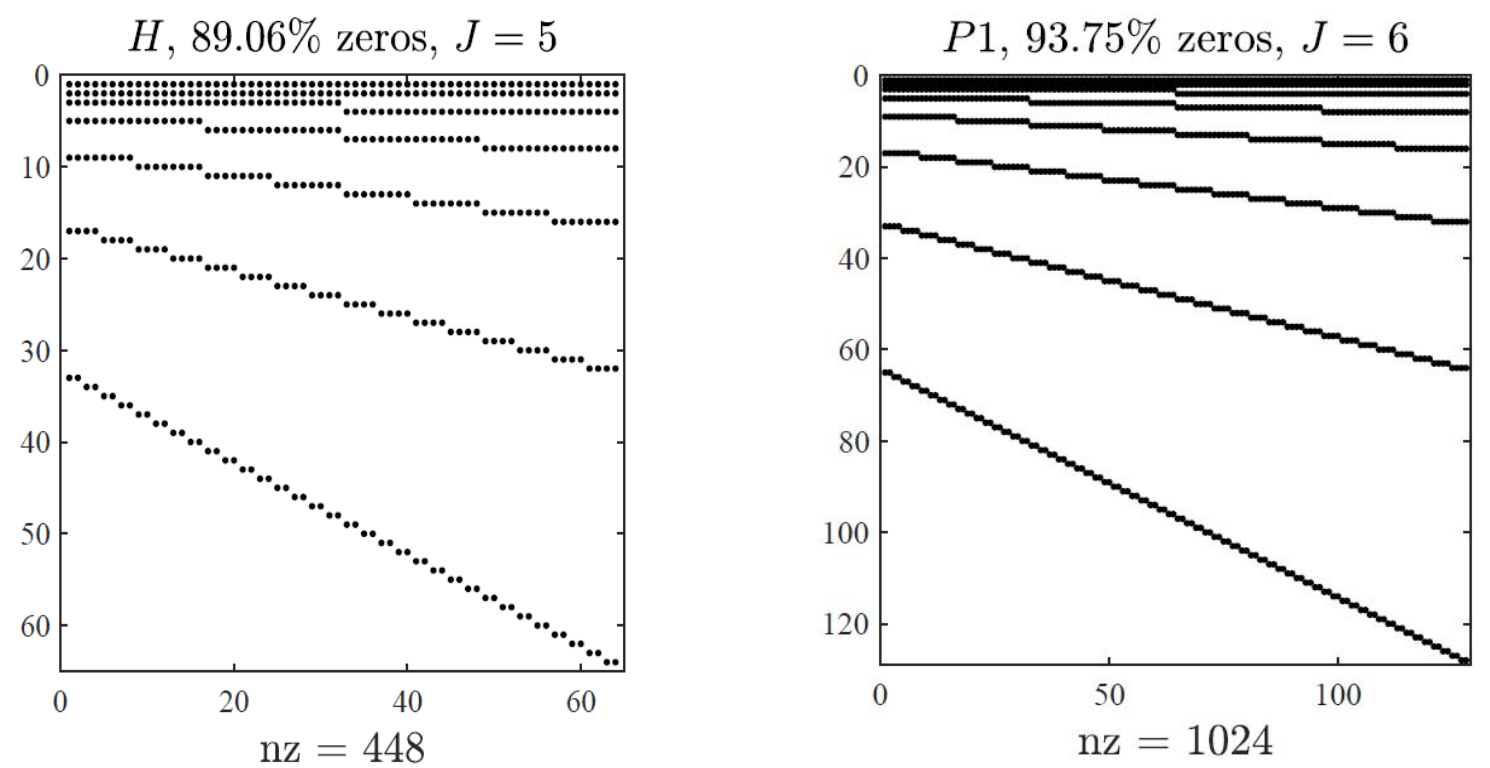

Fig.1. Nonzero structure of Haar matrix $H$ and its first integral $P 1$.

\section{Formulation of the problem}

The two-dimensional boundary-layer equations for the steady flow of an electrically conducting, viscous incompressible fluid in the presence of a magnetic field $B(x)$ normal to the stretching sheet are:

$$
\begin{aligned}
& \frac{\partial u}{\partial x}+\frac{\partial v}{\partial y}=0 \\
& u \frac{\partial u}{\partial x}+v \frac{\partial u}{\partial y}=v \frac{\partial^{2} u}{\partial y^{2}}-\frac{\sigma B^{2}(x)}{\rho} u
\end{aligned}
$$

subject to the boundary conditions

$$
\begin{array}{ll}
u=U(x), \quad v=0 & \text { at } \quad y=0, \\
u \rightarrow 0 \quad \text { as } \quad y \rightarrow \infty
\end{array}
$$

where $u$ and $v$ are the flow velocities in the $x$ and $y$ directions, respectively, $v, \rho$ and $\sigma$ are respectively, the kinematic viscosity, the fluid density and the electrical conductivity. The induced magnetic field, the external electric field and the electric field due to polarization of charges are presumed to be negligible.

It was proved that the similarity solutions exist when the non-uniform velocity of the stretching sheet is proportional to a power of the length coordinate, $x$, i.e., $U(x)=a x^{n}$ and the magnetic field $B(x)=B_{0} x^{(n-1) / 2}$, where $a, B_{0}$ and $n$ are parameters related to the surface stretching speed [8]. Introduce the similarity variables: 


$$
\psi=\sqrt{\frac{2 v x U(x)}{n+1}} f(\eta), \quad \eta=\sqrt{\frac{(n+1) U(x)}{2 v x}} y
$$

where $f(\eta)$ is a non-dimensional function related to the stream function $\psi(x, y)$ which is defined as

$$
(u, v)=\left(\frac{\partial \psi}{\partial y},-\frac{\partial \psi}{\partial x}\right)
$$

and $\eta$ is the non-dimensional variable. The horizontal and vertical velocity components are respectively:

$$
u=U(x) f^{\prime}(\eta), \quad v=\sqrt{\frac{v(n+1) U(x)}{2 x}}\left[\frac{n-1}{n+1} \eta f^{\prime}(\eta)+f(\eta)\right]
$$

By using the above transformations in Eqs (3.1)-(3.3), we obtain the third-order ordinary differential equation:

$$
f^{\prime \prime \prime}(\eta)+f(\eta) f^{\prime \prime}(\eta)-\beta f^{\prime}(\eta)^{2}-M f^{\prime}(\eta)=0
$$

with pertinent boundary conditions

$$
f(0)=0, \quad f^{\prime}(0)=1, \quad f^{\prime}(\infty)=0
$$

where

$$
\beta=\frac{2 n}{n+1}, \quad M=\frac{2 \sigma B_{0}^{2}}{\rho a(n+1)} .
$$

The system of Eqs (3.6)-(3.7) has exact analytic solutions for some special cases of $\beta[5,27]$

$$
\begin{aligned}
& f(\eta)=\sqrt{2} \tanh \left(\frac{\eta}{\sqrt{2}}\right) \quad \text { for } \quad \beta=-1, \quad M=0, \\
& f(\eta)=\frac{1-\exp (-\sqrt{1+M} \eta)}{\sqrt{1+M}} \quad \text { for } \quad \beta=1 .
\end{aligned}
$$

\section{Method of solution}

The semi-infinite physical domain of the problem Eq.(3.6) is truncated to finite domain by introducing an unknown finite boundary $\eta_{\infty}$ and normalized by introducing coordinate transformation $\xi=\eta / \eta_{\infty}$, so that all the boundary conditions of Eq.(3.6) are satisfied. Under this transformation Eq.(3.6) takes the form:

$$
f^{\prime \prime \prime}(\xi)+\eta_{\infty}^{2} f(\xi) f^{\prime \prime}(\xi)-\beta \eta_{\infty}^{2}\left(f^{\prime}(\xi)\right)^{2}-M \eta_{\infty}^{2} f^{\prime}(\xi)=0
$$


where $\eta_{\infty}$ is a sufficiently large value, which varies with flow parameters $\beta, M$ and is to be determined as a part of the solution procedure such that the third boundary condition in Eq.(3.7), $f^{\prime}\left(\eta_{\infty}\right)=0$ and the asymptotic condition, $f^{\prime \prime}\left(\eta_{\infty}\right)=0$ are satisfied. The corresponding boundary conditions Eq.(3.7) are

$$
f(0)=0, \quad f^{\prime}(0)=1, \quad f^{\prime}(1)=0 .
$$

\subsection{Numerical solution by HWCM}

The explicit analytical expression of Haar wavelets makes it possible to integrate Eq.(4.1) analytically an arbitrary number of times. With this observation, we seek the expressions for derivative functions $f^{\prime \prime \prime}(\xi)$ in Eq.(4.1) in terms of Haar series as follows:

$$
f^{\prime \prime}(\xi)=\sum_{i=1}^{2^{J+1}} a_{i} h_{i}(\xi)
$$

Using the boundary conditions Eq.(4.2) and integrating Eq.(4.3), we obtain the lower order derivatives:

$$
\begin{aligned}
& f^{\prime \prime}(\xi)=-1+\sum_{i=1}^{2^{J+1}} a_{i}\left[p_{1, i}(\xi)-C_{1, i}\right] \\
& f^{\prime}(\xi)=(1-\xi)+\sum_{i=1}^{2^{J+1}} a_{i}\left[p_{2, i}(\xi)-\xi C_{1, i}\right] \\
& f(\xi)=\xi\left(1-\frac{\xi}{2}\right)+\sum_{i=1}^{2^{J+1}} a_{i}\left[p_{3, i}(\xi)-\frac{\xi^{2}}{2} C_{1, i}\right]
\end{aligned}
$$

where $C_{1, i}=\int_{0}^{1} p_{1, i}(t) d t$. Substituting Eqs (4.3)-(4.6) in Eq.(4.1), we effectively transformed a two-point boundary value problem into a system of $2^{J+1}$ nonlinear equations in $2^{J+1}$ unknowns $a_{i}$ :

$$
F(A)=0
$$

where $F=\left(F_{1}(A), F_{2}(A), \ldots, F_{2}{ }^{J+1}(A)\right)$ and $A=\left(a_{1}, a_{2}, \ldots, a_{2^{J+1}}\right)$. Here, $F: R^{2^{J+1}} \rightarrow R^{2^{J+1}}$. We apply the collocation method to reduce this system into discrete form by introducing the collocation points

$$
\xi_{j}=(j-0.5) / 2^{J+1}, \quad j=1,2, \ldots, 2^{J+1}
$$

The nonlinear system Eq.(4.7) is solved by Newton's method for unknown coefficients $a_{i}$ and is given by

$$
A \leftarrow A-[J(A)]^{-1} F(A)
$$

where 


$$
\begin{aligned}
& F(A)=\sum_{i=1}^{2^{J+1}} a_{i} h_{i}\left(\xi_{j}\right)+\eta_{\infty}^{2}\left[\sum_{i=1}^{2^{J+1}} a_{i} p_{3, i}\left(\xi_{j}\right)-\frac{\xi_{j}^{2}}{2}-\frac{\xi_{j}^{2}}{2} \sum_{i=1}^{2^{J+1}} a_{i} C_{1, i}+\xi_{j}\right] \times \\
& \times\left[\sum_{i=1}^{2^{J+1}} a_{i} p_{1, i}\left(\xi_{j}\right)-1-\sum_{i=1}^{2^{J+1}} a_{i} C_{1, i}\right]-\beta \eta_{\infty}^{2}\left[\sum_{i=1}^{2^{J+1}} a_{i} p_{2, i}\left(\xi_{j}\right)-\xi_{j}-\xi_{j} \sum_{i=1}^{2^{J+1}} a_{i} C_{1, i}+1\right]^{2}+ \\
& -M \eta_{\infty}^{2}\left[\sum_{i=1}^{2^{J+1}} a_{i} p_{2, i}\left(\xi_{j}\right)-\xi_{j}-\xi_{j} \sum_{i=1}^{2^{J+1}} a_{i} C_{1, i}+1\right] ; \quad j=1,2, \ldots, 2^{J+1} \\
& J(A)=\left(\frac{\partial F}{\partial A}\right)_{2^{J+1} \times 2^{J+1}}
\end{aligned}
$$

The above equations are solved recursively using MATLAB. To initiate the procedure, we considered a nontrivial solution for initial resolution level $J$, which is then corrected by Newton's iterations. The wavelet coefficients for the next resolution level $J+1$ are evaluated as

$$
a_{i}^{(J+1)}= \begin{cases}a_{i}^{(J)} & \text { for } \quad i=1,2, \ldots, 2^{J}, \\ 0 & \text { for } \quad i=2^{J}+1,2^{J}+2, \ldots, 2^{J+1} .\end{cases}
$$

These approximations are corrected by Newton's method. The assumption Eq.(4.12) is based on the fact that higher coefficients of the sequence $a_{i}$ are relatively small. This assumption was totally justified in Section 5 (see, Figs $3 \mathrm{a}$ and $3 \mathrm{~b}$ ). The wall shear stress is evaluated by the explicit relation

$$
f^{\prime \prime}(0)=-1-\sum_{i=1}^{2^{J+1}} a_{i} C_{1, i}
$$

The results obtained for various flow parameters are shown in Tabs 3-5.

\subsection{Numerical solution by HWQLM}

In this method, we used the quasilinearization approach, introduced by Bellman and Kalaba [28], to linearize the problem Eq.(4.1):

$$
f^{\prime \prime \prime}(\xi)=G\left(f(\xi), f^{\prime}(\xi), f^{\prime \prime}(\xi), \xi\right)=-\eta_{\infty}^{2} f(\xi) f^{\prime \prime}(\xi)+\beta \eta_{\infty}^{2}\left(f^{\prime}(\xi)\right)^{2}+M \eta_{\infty}^{2} f^{\prime}(\xi)
$$

The linearized form of Eq.(4.14) is:

$$
\begin{aligned}
& f_{n+1}^{\prime \prime \prime}(\xi)=G\left(f_{n}(\xi), f_{n}^{\prime}(\xi), f_{n}^{\prime \prime}(\xi), \xi\right)+ \\
& +\sum_{j=0}^{2}\left(f_{n+1}^{(j)}(\xi)-f_{n}^{(j)}(\xi)\right) G_{f^{(j)}}\left(f_{n}(\xi), f_{n}^{\prime}(\xi), f_{n}^{\prime \prime}(\xi), \xi\right),
\end{aligned}
$$


which reduces to

$$
\begin{aligned}
& f_{n+1}^{\prime \prime \prime}(\xi)+\eta_{\infty}^{2} f_{n}(\xi) f_{n+1}^{\prime \prime}(\xi)-\left(2 \beta \eta_{\infty}^{2} f_{n}^{\prime}(\xi)+M \eta_{\infty}^{2}\right) f_{n+1}^{\prime}(\xi)+ \\
& +\eta_{\infty}^{2} f_{n}^{\prime \prime}(\xi) f_{n+1}(\xi)=-\beta \eta_{\infty}^{2}\left(f_{n}^{\prime}(\xi)\right)^{2}+\eta_{\infty}^{2} f_{n}^{\prime}(\xi) f_{n}^{\prime \prime}(\xi)
\end{aligned}
$$

with corresponding boundary conditions

$$
f_{n+1}(0)=0, \quad f_{n+1}^{\prime}(0)=1, \quad f_{n+1}^{\prime}(1)=0 .
$$

This method has a quadratic convergence. Applying the Haar wavelet method to Eq.(4.15) as explained in Section 4.1, we obtain the following system of $2^{J+1}$ linear equations:

$$
\begin{aligned}
& \sum_{i=1}^{2^{J+1}} a_{i}\left[h_{i}(\xi)+\eta_{\infty}^{2} f_{n}(\xi) p_{1, i}(\xi)-\eta_{\infty}^{2} f_{n}(\xi) C_{l, i}-\left(2 \beta \eta_{\infty}^{2} f_{n}^{\prime}(\xi)+M \eta_{\infty}^{2}\right) p_{2, i}(\xi)+\right. \\
& \left.+2 \beta \eta_{\infty}^{2} \xi f_{n}^{\prime}(\xi) C_{l, i}+M \eta_{\infty}^{2} \xi C_{l, i}+\eta_{\infty}^{2} f_{n}^{\prime \prime}(\xi) p_{3, i}(\xi)-\frac{\eta_{\infty}^{2}}{2} \xi^{2} f_{n}^{\prime \prime}(\xi) C_{l, i}\right]= \\
& =\eta_{\infty}^{2} f_{n}(\xi)-2 \beta \eta_{\infty}^{2} \xi f_{n}^{\prime}(\xi)-M \eta_{\infty}^{2} \xi+2 \beta \eta_{\infty}^{2} f_{n}^{\prime}(\xi)+M \eta_{\infty}^{2}+ \\
& +\frac{\eta_{\infty}^{2}}{2} \xi^{2} f_{n}^{\prime \prime}(\xi)-\eta_{\infty}^{2} \xi f_{n}^{\prime \prime}(\xi)-\beta \eta_{\infty}^{2}\left(f_{n}^{\prime}(\xi)\right)^{2}+\eta_{\infty}^{2} f_{n}(\xi) f_{n}^{\prime \prime}(\xi) .
\end{aligned}
$$

With a suitable initial approximation, the system Eq.(4.17) is solved for Haar coefficients $a_{i}$. MATLAB program is developed to find the wavelet solution of the problem at various levels of resolution.

\subsection{Error analysis}

The convergence and efficiency of the Haar wavelet method have been studied by Majak et al. [29] and they proved that for the Haar wavelet approximation, the error is proportional to the reciprocal of the level of resolution. In our study, we estimate the exactness of the obtained wavelet results by estimating $L_{\infty}$ errors as follows:

$$
\delta_{J}=\max _{1 \leq j \leq 2^{J+1}}\left|f\left(\eta_{j}\right)^{\text {exact }}-f\left(\eta_{j}\right)^{\text {num }}\right|
$$

For the case of $\beta$ where exact solutions are not known, we compute the solution error using the relation

$$
\Delta_{J}=\left|\frac{\varepsilon_{J+1}-\varepsilon_{J}}{\varepsilon_{J}}\right|
$$

where

$$
\varepsilon_{J}=\left(\frac{1}{2^{J+1}}\right) \sum_{j=1}^{2^{J+1}}\left|f_{J}\left(\eta_{j}\right)\right| ; \quad j=1,2, \ldots, 2^{J+1}
$$


$f_{J}(\eta)$ is the solution of Eq.(3.6) for the level of resolution $J$. The convergence rate of the solution of Eq.(3.6) is computed by the ratio given below

$$
\sigma_{J}=\frac{\Delta_{J-1}}{\Delta_{J}} ; \quad J=2,3, \ldots
$$

The $L_{\infty}$ errors and the rate of convergence at various resolution levels for $\beta=-1,1,1.5 \& 5$ are computed and presented in Tabs 1-2 and Fig. 2.

\section{Results and discussion}

The problem of an MHD boundary-layer flow due to a stretching sheet is studied using novel wavelet based numerical schemes. The motion of the fluid is governed by nonlinear ODE Eq.(3.6) with boundary conditions Eq.(3.7). The governing problem Eq.(3.6) is solved using HWCM by normalizing the semi-infinite domain with suitable coordinate transformation. The expressions for velocity profiles and the shear stress are given by the relations Eq.(4.5) and Eq.(4.13), respectively. Numerical computations are performed for different values of physical parameters $M$ and $\beta$ at various resolution levels.

Table 1. Error estimates of solution of Eq.(3.6) using HWCM for $\beta=-1,1$ at different resolution levels.

\begin{tabular}{lccc}
\hline$J$ & $\delta_{J}$ for $\beta=-1$ & \multicolumn{2}{c}{$\delta_{J}$ for $\beta=1$} \\
\cline { 2 - 4 } & $(M=0)$ & $M=5$ & $M=10$ \\
\hline 1 & $1.343078 E-01$ & $7.941058 E-02$ & $5.761444 E-02$ \\
2 & $1.706404 E-01$ & $2.745206 E-02$ & $1.729623 E-02$ \\
3 & $2.011636 E-02$ & $9.469628 E-03$ & $6.202398 E-03$ \\
4 & $4.613047 E-03$ & $2.620338 E-03$ & $1.724250 E-03$ \\
5 & $1.127123 E-03$ & $6.734131 E-04$ & $4.433600 E-04$ \\
6 & $2.798058 E-04$ & $1.695584 E-04$ & $1.116489 E-04$ \\
7 & $6.909750 E-05$ & $4.247781 E-05$ & $2.797759 E-05$ \\
8 & $1.651685 E-05$ & $1.063719 E-05$ & $7.013003 E-06$ \\
9 & $5.731338 E-06$ & $2.672670 E-06$ & $1.768994 E-06$ \\
10 & $1.483911 E-06$ & $6.812946 E-07$ & $4.578467 E-07$ \\
\hline
\end{tabular}

To confirm the accuracy and validity of the results obtained, the problem is also analysed by HWQLM. The physical dynamics of the problem is analysed through the velocity profiles and shear stress for different parameters. In the absence of an applied magnetic field, i.e., $M=0$ and general $\beta$ the problem Eq.(3.6) reduces to the study of a two-dimensional laminar flow due to a stretching wall $[10,30,31]$. Also, for $\beta=0$ the governing problem describes the well-known Sakiadis problem [32]. We have estimated $L_{\infty}$ errors for $\beta=-1$ and $\beta=1$ values using the relation Eq.(4.18) and they are given in Tab.1. It is evident from the table that the error decreases as the level of resolution increases. We also computed solution errors and rate of convergence for $\beta=1.5$ and $\beta=5$ where the analytical solutions are not available. The results obtained are given in Tab.2. and Fig.2. at different levels of resolution. The nature of the errors obtained in these cases is similar to the previous one (cf. see Tab.1.). Clearly, the error bound for Haar wavelet approximation is inversely proportional to the resolution level. Also, it is observed from Tab.2. that by doubling the number of collocation points the error roughly decreases four times as $J$ increases, i.e., the rate of convergence for the Haar wavelet series is 


$$
O\left[\left(\frac{1}{2^{J+1}}\right)^{2}\right]
$$

This numerical estimation of $\sigma_{J}$ ultimately demonstrates the convergence and stability of the proposed method. A similar trend is noticed for HWQLM results also, but not included here.

Table 2. Error estimates and rate of convergence of solution of Eq.(3.6) using HWCM for $\beta=1.5$ at different resolution levels.

\begin{tabular}{cccccc}
\hline$J$ & \multicolumn{2}{c}{$M=5$} & & \multicolumn{2}{c}{$M=10$} \\
\cline { 2 - 3 } \cline { 5 - 6 } & $\Delta_{J}$ & $\sigma_{J}$ & & $\Delta_{J}$ & $\sigma_{J}$ \\
\hline 1 & $4.106421 E-02$ & - & & $5.857679 E-02$ & - \\
2 & $2.659896 E-02$ & 1.54383 & & $2.082747 E-02$ & 2.81248 \\
3 & $1.396369 E-02$ & 1.90487 & & $1.187476 E-02$ & 1.75393 \\
4 & $4.267115 E-03$ & 3.27240 & & $3.613376 E-03$ & 3.28633 \\
5 & $1.125336 E-03$ & 3.79186 & & $9.498328 E-04$ & 3.80422 \\
6 & $2.851973 E-04$ & 3.94581 & & $2.404813 E-04$ & 3.94972 \\
7 & $7.154423 E-05$ & 3.98631 & & $6.031122 E-05$ & 3.98734 \\
8 & $1.790142 E-05$ & 3.99657 & & $1.508977 E-05$ & 3.99683 \\
9 & $4.476315 E-06$ & 3.99914 & & $3.773190 E-06$ & 3.99921 \\
\hline
\end{tabular}

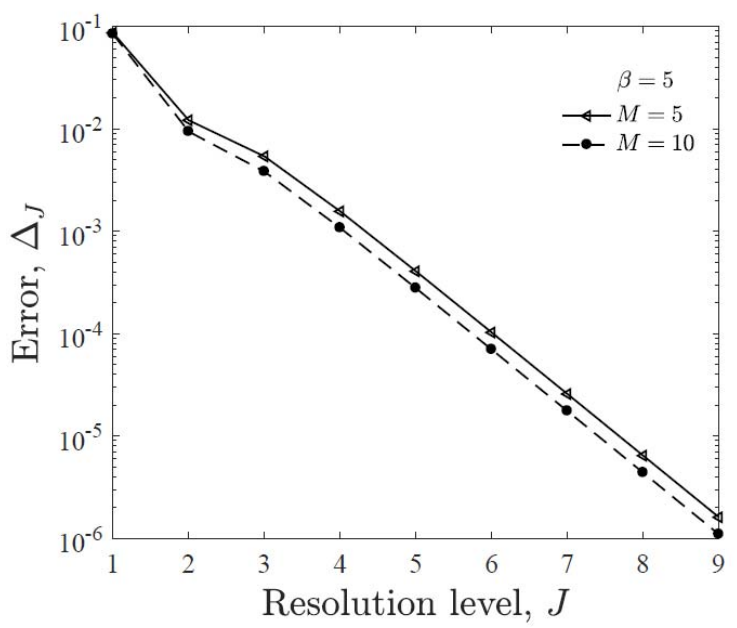

Fig.2. Error estimates in the solution of Eq.(3.6) using HWCM for $\beta=5$.

From Figs $3 a$ and $3 b$, it is interesting to note that, the wavelet coefficients are in direct relation with level of resolution. That is, for increase in $J$, the number of coefficients $a_{i}$ also increases by $2^{J+1}$. By increasing the wavelet number $i$, the wavelet coefficients $a_{i}$ rapidly decreases with higher coefficients values attains to zero and also, the values of $a_{i}$ are significantly smaller, which eventually provides high accuracy solutions (i.e., high rate of convergence) with finite number of Haar coefficients. Figure 4 describes the wavelet solution $f(\eta)$ at different iterations so as to corroborate the speed of convergence of HWQLM. It can be seen 
from the graph that the first few iterations of HWQLM itself provide very accurate and numerically stable answers.
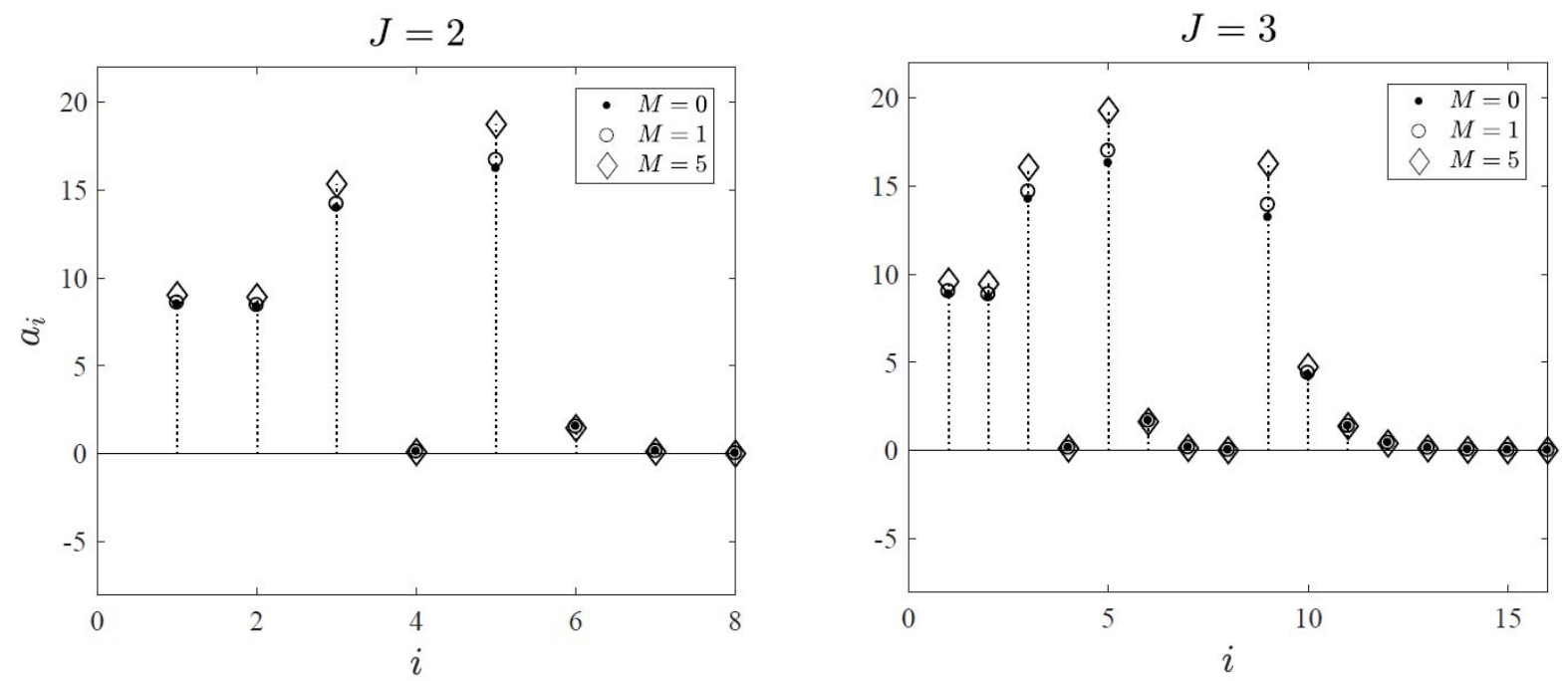

Fig.3a. Values of Haar wavelet coefficients for solution of Eq.(3.6) for $\beta=1$ at resolution levels $J=2$ and 3 .
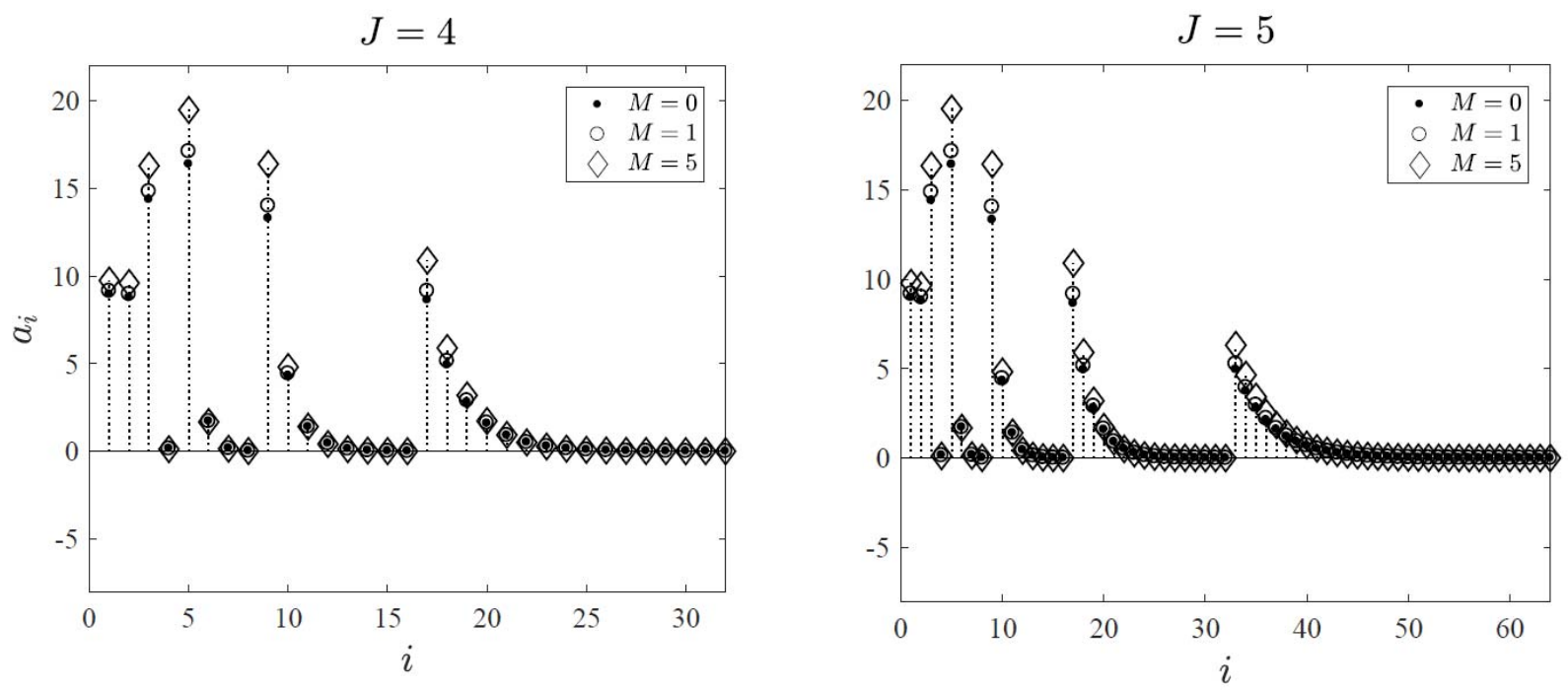

Fig.3b. Values of Haar wavelet coefficients for solution of Eq.(3.6) for $\beta=1$ at resolution levels $J=4$ and 5 .

The influence of the magnetic parameter $M$ and $\beta$ on the wall shear stress $f^{\prime \prime}(0)$ is presented in Tabs 3-5 (cf. Eq.(4.13)). The results are in close agreement for all the solutions obtained and giving better accuracy for a higher resolution level. The computational time efficiency of the proposed methods has been explicitly calculated by employing timing command and shown in Tab.6. for specific values of flow parameters. Intel Core i3 processor with 4GB RAM is used for the calculation. It must be emphasized that the amount of computational effort is significantly less in the repeated methods. The comparison of solution $f(\eta)$ obtained by HWCM and HWQLM with exact solution is shown in Fig.5a. It is important to note that unlike the shooting method reported in [8], the wavelet method does not require initial guess for $f^{\prime \prime}(0)$ at each and every case corresponding to parameters $\beta$ and $M$. The assumption taken in Eq.(4.12) by itself works well for all the 
considered cases of $\beta$ and $M$, which enhances the effectiveness of the proposed methods. The velocity profiles for various values of the magnetic parameter $M$ and $\beta=1$ using HWQLM and HWCM are plotted in Fig.5b. It is observed that the boundary-layer becomes thinner when the magnetic parameter $M$ increases and this causes an increase in the shear stress (in absolute sense). Also, the domain of the problem becomes smaller and smaller.
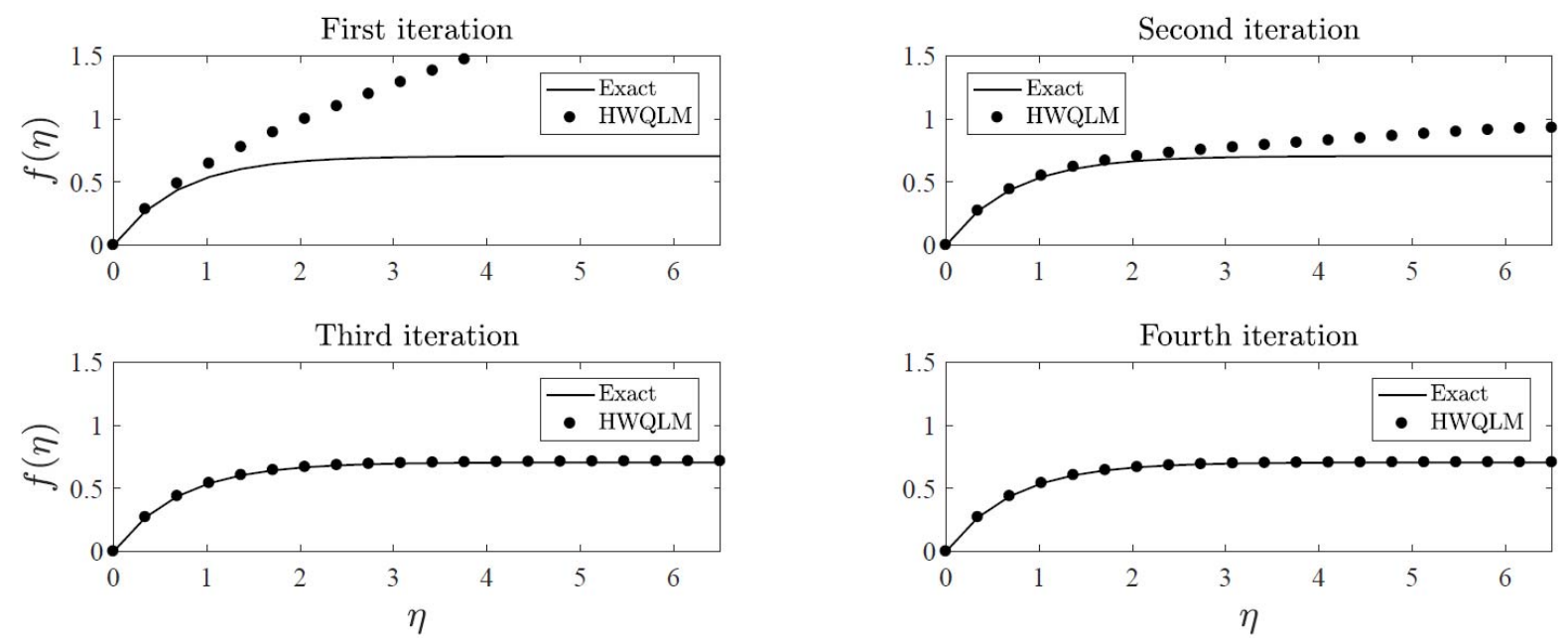

Fig.4. Comparison of analytical solution and HWQLM solution for different iterations (resolution level $J=6$ and $M=\beta=1)$.

Table 3. Comparison of values of the skin friction coefficient $f^{\prime \prime}(0)$ for $\beta=1$ and various values of the magnetic parameter $M$.

\begin{tabular}{|c|c|c|c|c|c|}
\hline \multirow{3}{*}{$M$} & \multicolumn{5}{|c|}{$\beta=1$} \\
\hline & \multicolumn{2}{|c|}{$H W Q L M$} & \multicolumn{2}{|c|}{ HWCM } & \multirow{2}{*}{$\begin{array}{c}\text { Exact } \\
\text { solution [5] }\end{array}$} \\
\hline & $J=9$ & $J=10$ & $J=9$ & $J=10$ & \\
\hline 0 & -1.00000 & -1.00000 & -1.00000 & -1.00000 & -1.00000 \\
\hline 1 & -1.41421 & -1.41421 & -1.41421 & -1.41421 & -1.41421 \\
\hline 5 & -2.44949 & -2.44948 & -2.44949 & -2.44948 & -2.44948 \\
\hline 10 & -3.31662 & -3.31662 & -3.31662 & -3.31662 & -3.31662 \\
\hline 50 & -7.14142 & -7.14142 & -7.14142 & -7.14142 & -7.14142 \\
\hline 100 & -10.04987 & -10.04987 & -10.04987 & -10.04987 & -10.04987 \\
\hline 500 & -22.38303 & -22.38302 & -22.38303 & -22.38302 & -22.38302 \\
\hline 1000 & -31.63858 & -31.63858 & -31.63858 & -31.63858 & -31.63858 \\
\hline 1500 & -38.74274 & -38.74274 & -38.74274 & -38.74274 & -38.74274 \\
\hline 2000 & -44.73253 & -44.73253 & -44.73253 & -44.73253 & -44.73253 \\
\hline$\downarrow$ & & & & & \\
\hline
\end{tabular}


Table 4. Comparison of values of the skin friction coefficient $f^{\prime \prime}(0)$ for $\beta=-1.0,-1.5$ and different values of the magnetic parameter $M$ (Resolution level $J=9$ ).

\begin{tabular}{|c|c|c|c|c|c|c|}
\hline$\beta$ & $M$ & HWQLM & HWCM & $\begin{array}{l}\text { Shooting } \\
\text { method }[8]\end{array}$ & $\begin{array}{l}\text { Crocco } \\
\text { transformation } \\
\text { method }[8]\end{array}$ & ADM [11] \\
\hline \multirow[t]{15}{*}{-1.0} & 0 & 0.0 & 0.0 & 0.0 & -0.31345 & 0.0 \\
\hline & 0.1 & -0.13215 & -0.13215 & -0.13215 & -0.18116 & - \\
\hline & 0.2 & -0.24783 & -0.24783 & -0.24783 & -0.19294 & - \\
\hline & 0.3 & -0.35006 & -0.35006 & -0.35006 & -0.33459 & - \\
\hline & 0.4 & -0.44140 & -0.44140 & -0.44140 & -0.43711 & - \\
\hline & 0.5 & -0.52395 & -0.52395 & -0.52395 & -0.52349 & - \\
\hline & 1 & -0.85111 & -0.85111 & -0.85111 & -0.85210 & -0.8511 \\
\hline & 5 & -2.16287 & -2.16287 & -2.16287 & -2.16289 & -2.1628 \\
\hline & 10 & -3.11003 & -3.11003 & -3.11003 & -3.11003 & -3.1100 \\
\hline & 50 & -7.04756 & -7.04756 & - & - & -7.0475 \\
\hline & 100 & -9.98335 & -9.98335 & -9.98335 & -9.98335 & -9.9833 \\
\hline & 500 & -22.35326 & -22.35326 & - & - & -22.3532 \\
\hline & 1000 & -31.61754 & -31.61754 & - & - & -31.6175 \\
\hline & 1500 & -38.72558 & -38.72558 & - & - & - \\
\hline & 2000 & -44.71768 & -44.71768 & - & - & - \\
\hline \multirow[t]{14}{*}{-1.5} & 0 & 0.72725 & 0.72725 & 0.72725 & 0.55680 & $\begin{array}{l}\text { Solution } \\
\text { diverges }\end{array}$ \\
\hline & 0.1 & 0.45107 & 0.45107 & 0.45107 & 0.50285 & - \\
\hline & 0.2 & 0.23038 & 0.23038 & 0.23038 & 0.43877 & - \\
\hline & 0.3 & 0.05203 & 0.05203 & 0.05203 & 0.35849 & - \\
\hline & 0.4 & -0.09506 & -0.09506 & -0.09506 & -0.24592 & - \\
\hline & 0.5 & -0.21922 & -0.21922 & -0.21922 & -0.10844 & - \\
\hline & 1 & -0.65298 & -0.65298 & -0.65298 & -0.65325 & -0.6532 \\
\hline & 5 & -2.08524 & -2.08524 & -2.08524 & -2.08528 & -2.0852 \\
\hline & 10 & -3.05623 & -3.05623 & -3.05623 & -3.05623 & -3.0562 \\
\hline & 50 & -7.02387 & -7.02387 & - & - & -7.0238 \\
\hline & 100 & -9.96665 & -9.96665 & -9.96665 & -9.96665 & -9.9666 \\
\hline & 500 & -22.34579 & -22.34579 & - & - & -22.3457 \\
\hline & 1000 & -31.61227 & -31.61227 & - & - & -31.6122 \\
\hline & 1500 & -38.72127 & -38.72127 & - & - & - \\
\hline$\downarrow$ & 2000 & -44.71396 & -44.71396 & - & - & - \\
\hline
\end{tabular}

Figures $6 \mathrm{a}$ and $7 \mathrm{a}$ present a comparison between the HWQLM and the HWCM solutions $f(\eta)$ at $\beta=1.5,-1.5$. The variations of $f^{\prime}(\eta)$ with $\eta$ approximated by the proposed methods are depicted in Figs $6 \mathrm{~b}$ and $7 \mathrm{~b}$. Table 4 shows that Adomian decomposition method fails to give converging values for $f^{\prime \prime}(0)$ at $\beta=-1.5$ and $M$ about 1 , which is clearly highlighted in the earlier findings [11]. A significant change in the nature of the flow occurs at $M \leq 0.5$ and for a negative nonlinear stretching parameter $\beta$. This can be observed from Fig. 7b. that for the values of $M \leq 0.5, f^{\prime}(\eta)$ behaves differently near the surface. That is, $f^{\prime}(\eta)$ attains a maximum before it starts to decrease. The methods described in [11, 12] fail to model this behaviour properly. However, our proposed wavelet methods persists to produce the desired solution regardless of whether the 
magnetic parameter $\mathrm{M}$ is smaller or larger without any convergence issues. Also, the results obtained by these methods are more accurate and giving converging solutions than the homotopy analysis method [33]. The variations of velocity and shear stress profiles for different values of $M$ and $\beta=-1.5,-1.0,5.0$ are plotted in Figs 8-10. An interesting variation behaviour in shear stress profiles is found in Figs $8,9 \mathrm{~b}$ for $\beta<0$ and smaller values of $M$ wherein $f^{\prime \prime}(\eta)$ decreases before satisfying the asymptotic condition $f^{\prime \prime}\left(\eta_{\infty}\right)=0$.

Table 5. Comparison of values of the skin friction coefficient $f^{\prime \prime}(0)$ for $\beta=1.5,5$ and different values of the magnetic parameter $M$ (Resolution level $J=9$ ).

\begin{tabular}{|c|c|c|c|c|c|c|}
\hline$\beta$ & $M$ & HWQLM & HWCM & $\begin{array}{l}\text { Shooting } \\
\text { method }[8]\end{array}$ & $\begin{array}{l}\text { Crocco } \\
\text { transformation } \\
\text { method [8] }\end{array}$ & ADM [11] \\
\hline \multirow{10}{*}{1.5} & 0 & -1.14860 & -1.14860 & -1.14860 & -1.14902 & -1.1547 \\
\hline & 1 & -1.52527 & -1.52527 & -1.52527 & -1.52533 & -1.5252 \\
\hline & 5 & -2.51615 & -2.51615 & -2.51615 & -2.51616 & -2.5161 \\
\hline & 10 & -3.36631 & -3.36631 & -3.36631 & -3.36632 & -3.3663 \\
\hline & 50 & -7.16471 & -7.16471 & -7.16471 & -7.16471 & -7.1647 \\
\hline & 100 & -10.06645 & -10.06645 & -10.0664 & -10.0664 & -10.0776 \\
\hline & 500 & -22.39049 & -22.39049 & - & - & -22.3904 \\
\hline & 1000 & -31.64388 & -31.64388 & - & - & -31.6438 \\
\hline & 1500 & -38.74708 & -38.74708 & - & - & - \\
\hline & 2000 & -44.73631 & -44.73631 & - & - & - \\
\hline \multirow[t]{10}{*}{5.0} & 0 & -1.90253 & -1.90253 & -1.90253 & -1.90433 & -1.9098 \\
\hline & 1 & -2.15290 & -2.15290 & -2.15290 & -2.15344 & -2.1528 \\
\hline & 5 & -2.94144 & -2.94144 & -2.94144 & -2.94150 & -2.9414 \\
\hline & 10 & -3.69566 & -3.69566 & -3.69566 & -3.69567 & -3.6956 \\
\hline & 50 & -7.32561 & -7.32561 & -7.32561 & -7.32561 & -7.3256 \\
\hline & 100 & -10.18167 & -10.18167 & -10.1816 & -10.1816 & -10.1816 \\
\hline & 500 & -22.44255 & -22.44255 & - & - & -22.4425 \\
\hline & 1000 & -31.68074 & -31.68074 & - & - & -31.6806 \\
\hline & 1500 & -38.77718 & -38.77718 & - & - & - \\
\hline & 2000 & -44.76238 & -44.76238 & - & - & - \\
\hline
\end{tabular}

Table 6. The CPU time taken to obtain the wavelet solution of Eq.(3.6) for $\beta=1$ and $M=5$.

\begin{tabular}{cccc}
\hline $\begin{array}{c}\text { Resolution level } \\
J\end{array}$ & $\begin{array}{c}\text { No. of collocation } \\
\text { points }\end{array}$ & \multicolumn{2}{c}{ Time in Seconds } \\
\cline { 3 - 4 } & & HWCM & $\begin{array}{c}\text { HWQLM } \\
\text { (4 iterations) }\end{array}$ \\
\hline 4 & 32 & 0.21 & 0.09 \\
5 & 64 & 0.25 & 0.13 \\
6 & 128 & 0.38 & 0.18 \\
7 & 256 & 0.59 & 0.32 \\
8 & 512 & 1.04 & 0.55 \\
9 & 1024 & 5.95 & 4.09 \\
\hline
\end{tabular}



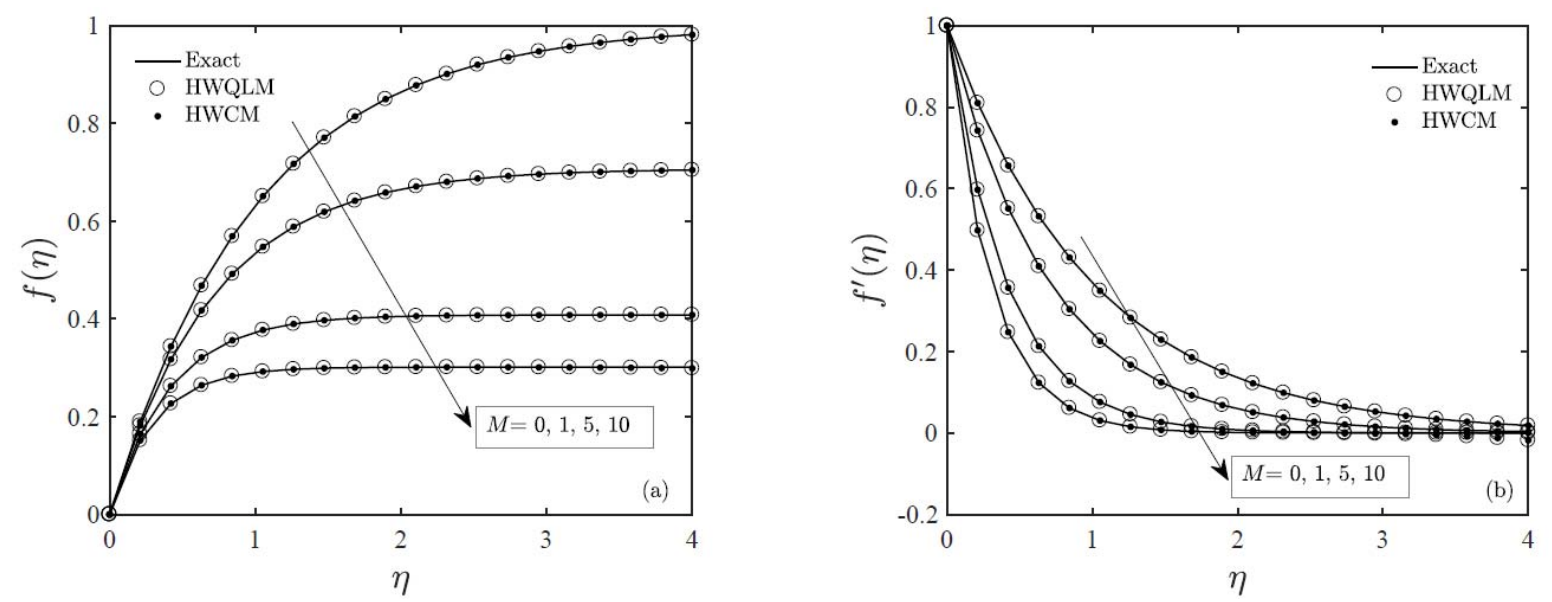

Fig.5. Comparison of (a) solution $f(\eta)$ and (b) velocity $f^{\prime}(\eta)$ of Eq.(3.6) obtained by HWCM and HWQLM with the analytical solution for $\beta=1$.
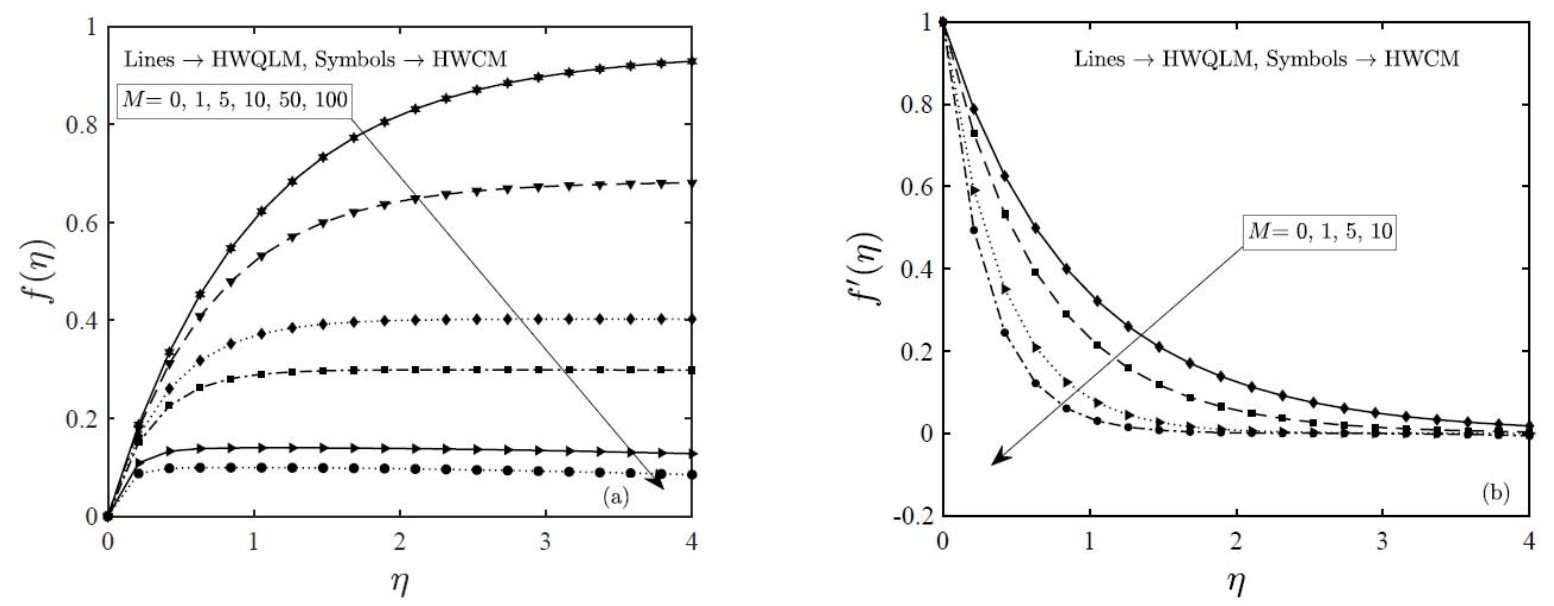

Fig.6. Comparison of (a) solution $f(\eta)$ and (b) velocity $f^{\prime}(\eta)$ of Eq.(3.6) obtained by HWCM and HWQLM with the analytical solution for $\beta=1.5$.
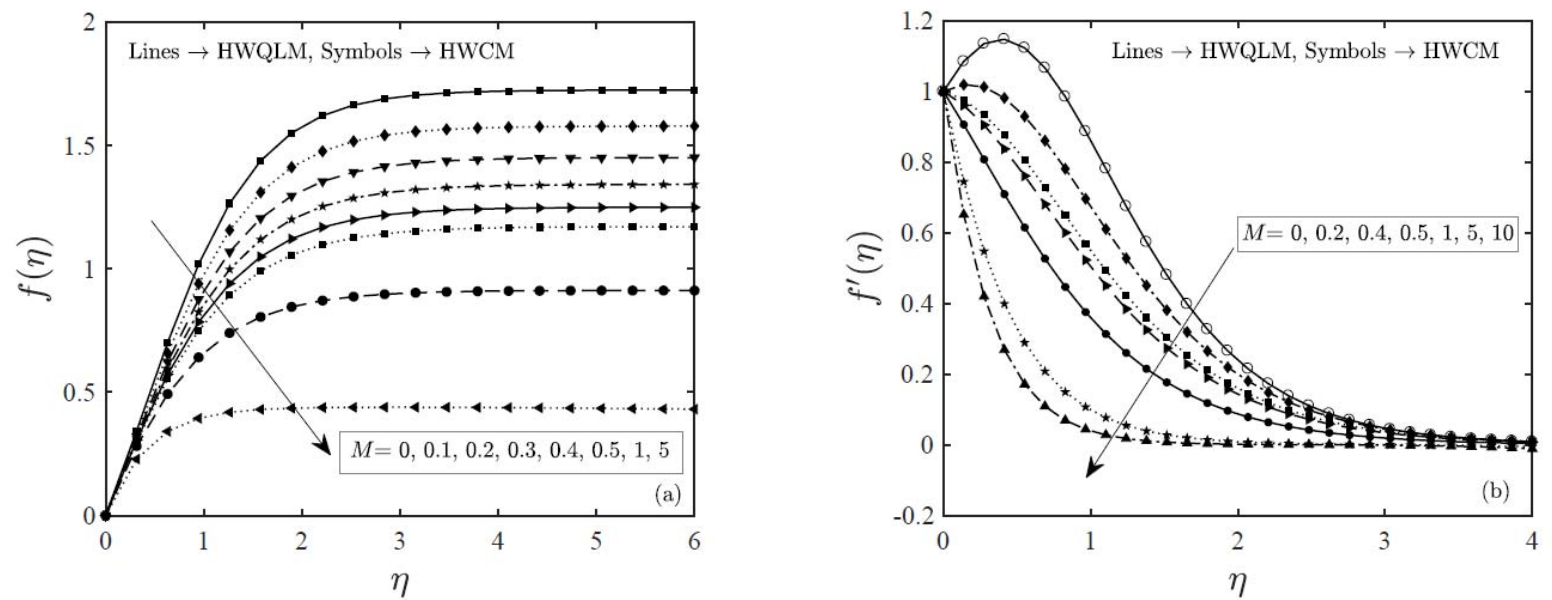

Fig.7. Comparison of (a) solution $f(\eta)$ and (b) velocity $f^{\prime}(\eta)$ of Eq.(3.6) obtained by HWCM and HWQLM with the analytical solution for $\beta=-1.5$. 


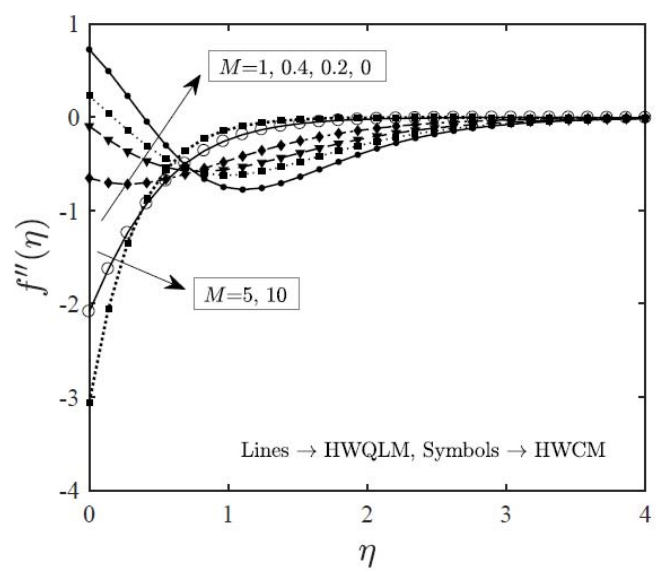

Fig.8. Shear stress profiles in the boundary-layers under different values of $M$ and $\beta=-1.5$.
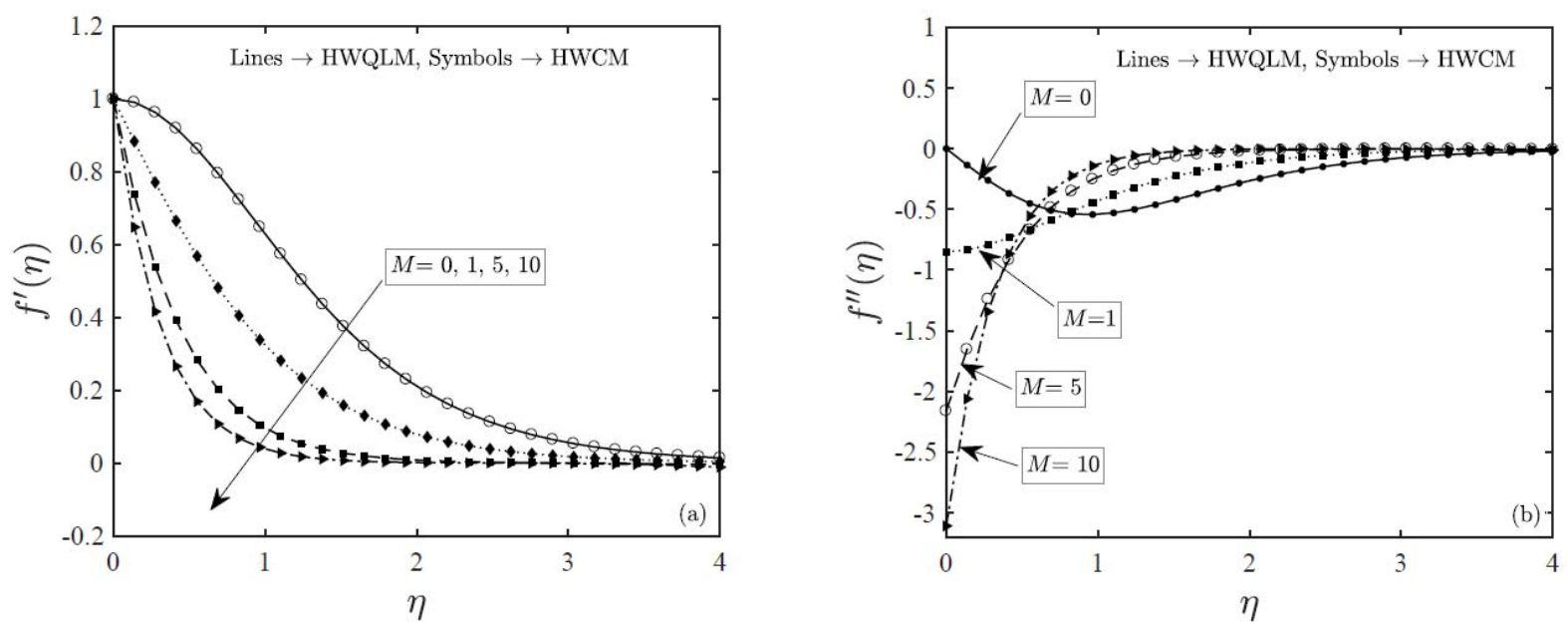

Fig.9. Velocity and shear stress profiles in the boundary-layers under different values of $M$ and $\beta=-1.0$.

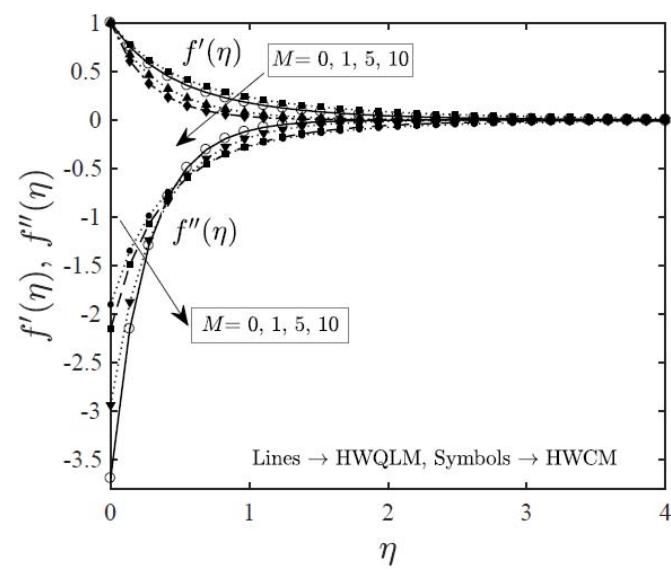

Fig.10. Velocity and shear stress profiles in the boundary-layers under different values of $M$ and $\beta=5.0$. 


\section{Conclusion}

We presented Haar wavelet based numerical schemes to investigate an MHD boundary-layer flow due to a nonlinear stretching sheet. The flow is governed by the Falkner-Skan family of equations. The advantage of the proposed methods over semi-analytical and numerical methods is that they work effectively for all values of $\beta$ and $M$ without encountering any difficulty. The results obtained in this paper confirm that these methods prove to be a competitive alternative to existing semi-analytical and traditional numerical methods. The methods guarantee the convergence of the solution and it can be easily employed to many nonlinear boundary value problems with semi-infinite or infinite domains arising in fluid mechanics.

\section{Acknowledgements}

Authors thank the anonymous reviewers for their educative comments which led to the present form.

\section{Nomenclature}

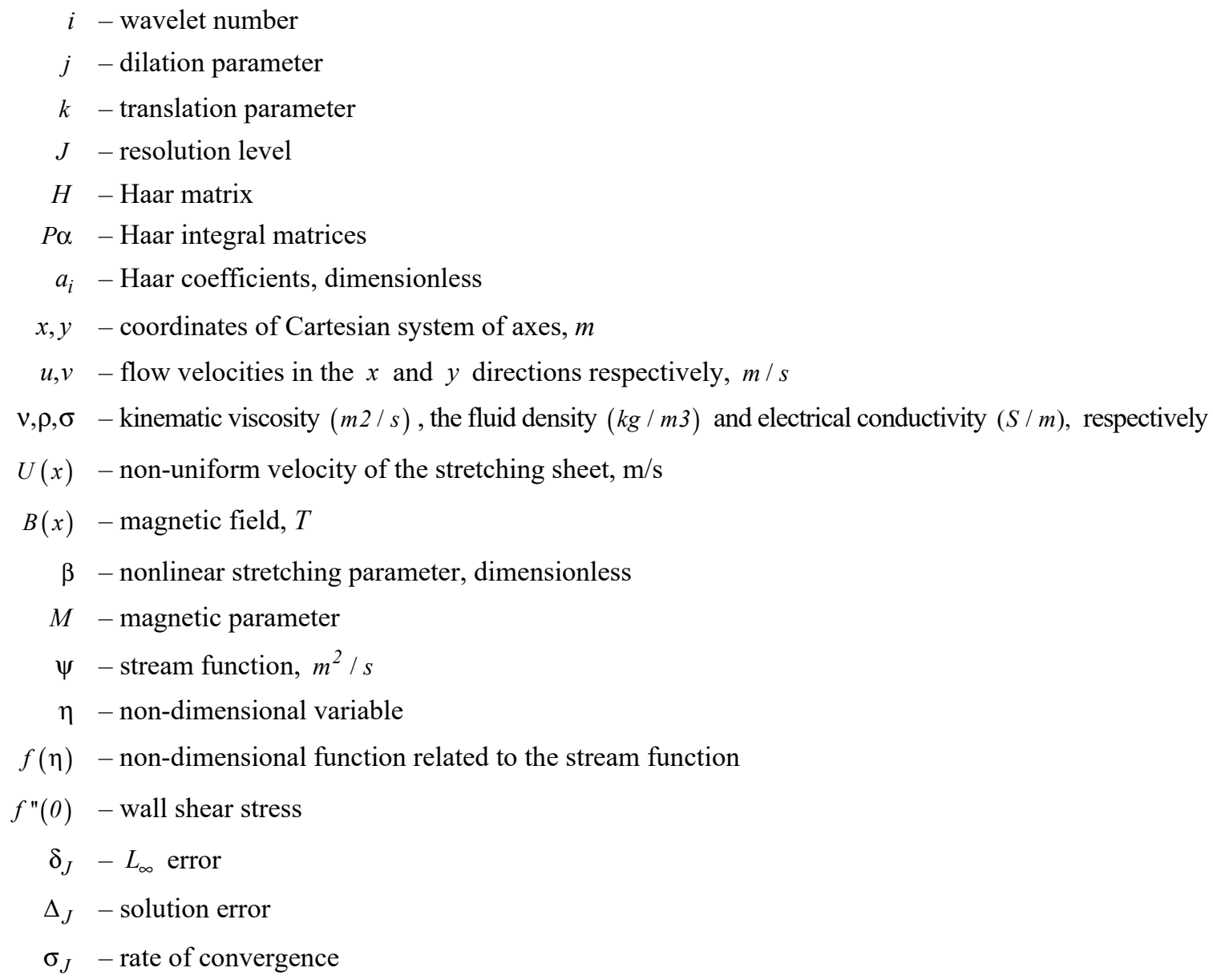

\section{References}

[1] Rashad A.M., Rashidi M.M., Giulio Lorenzini, Sameh E.A. and Abdelraheem M.A. (2017): Magnetic field and internal heat generation effects on the free convection in a rectangular cavity filled with a porous medium saturated with Cu-water nanofluid.- International Journal of Heat and Mass Transfer, vol.104, pp.878-889. 
[2] Bhatti M.M., Mishra S.R., Abbas T. and Rashidi M.M. (2018): A mathematical model of MHD nanofluid flow having gyrotactic microorganisms with thermal radiation and chemical reaction effects.- Neural Computing and Applications, vol.30, pp.1237-1249.

[3] Mansoury D., Doshmanziari F.I., Rezaie S. and Rashidi M.M. (2019): Effect of $\mathrm{Al}_{2} \mathrm{O}_{3}$ /water nanofluid on performance of parallel flow heat exchangers.- Journal of Thermal Analysis and Calorimetry, vol.135, pp.625-643.

[4] Crane L.J. (1970): Flow past a stretching plate.- Zeitschrift für angewandte Mathematik und Physik ZAMP, vol.21, pp.645-647.

[5] Chakrabarti A. and Gupta A.S. (1979): Hydromagnetic flow and heat transfer over a stretching sheet.- Quarterly of Applied Mathematics, vol.37, pp.73-78.

[6] Banks W.H.H. and Zaturska M.B. (1986): Eigen solutions in boundary-layer flow adjacent to a stretching wall.IMA. J. Appl. Math., vol.36, pp.263-273.

[7] Afzal N. (1993): Heat transfer from a stretching surface.- International Journal of Heat and Mass Transfer, vol.36, pp. 1128-1131.

[8] Chiam T.C. (1995): Hydromagnetic flow over a surface stretching with a power-law velocity.- International Journal of Engineering Science, vol.33, pp.429-435.

[9] Liao S. (2005): A new branch of solutions of boundary-layer flows over an impermeable stretched plate.- International Journal of Heat and Mass Transfer, vol.48, pp.2529-2539.

[10] Kudenatti R.B. (2012): A new exact solution for boundary layer flow over a stretching plate.- International Journal of Non-Linear Mechanics, vol.47, pp.727-733.

[11] Hayat T., Hussain Q. and Javed T. (2009): The modified decomposition method and Padè approximants for the MHD flow over a non-linear stretching sheet.- Nonlinear Analysis: Real World Applications, vol.10, pp.966-973.

[12] Rashidi M.M. (2009): The modified differential transform method for solving MHD boundary-layer equations.Computer Physics Communications, vol.180, pp.2210-2217.

[13] Mehmood A., Munawar S. and Ali A. (2010): Comments to: Homotopy analysis method for solving the MHD flow over a non-linear stretching sheet (Commun. Nonlinear Sci. Numer. Simul. 14(2009)2653-2663).-Communications in Nonlinear Science and Numerical Simulation, vol.15, pp.4233-4240.

[14] Chen C.F. and Hsiao C.H. (1997): Haar wavelet method for solving lumped and distributed-parameter systems.IEE Proc.-Control Theory Appl., vol.144, pp.87-94.

[15] Lepik Ü. (2006): Haar wavelet method for nonlinear integro-differential equations.- Applied Mathematics and Computation, vol.176, pp.324-333.

[16] Lepik Ü. (2007): Application of the Haar wavelet transform to solving integral and differential equations.Proceedings-Estonian Academy of Sciences Physics Mathematics, vol.56, pp.28-46.

[17] Lepik Ü. (2008): Haar wavelet method for solving higher order differential equations.- International Journal of Mathematics and Computation, vol.8, pp.84-94.

[18] Lepik Ü. (2009): Solving fractional integral equations by the Haar wavelet method.- Applied Mathematics and Computation, vol.214, pp.468-478.

[19] Bujurke N.M., Salimath C.S. and Shiralashetti S.C. (2008): Numerical solution of stiff systems from nonlinear dynamics using single-term Haar wavelet series.- Nonlinear Dynamics, vol.51, pp.595-605.

[20] Hariharan G. and Kannan K. (2010): Haar wavelet method for solving some nonlinear Parabolic equations -Journal of Mathematical Chemistry, vol.48, pp.1044-1061.

[21] Kanth A.S.V.R. and Kumar N.U. (2013): A Haar wavelet study on convective-radiative fin under continuous motion with temperature-dependent thermal conductivity.- Walailak Journal of Science and Technology, vol.11, pp.211224.

[22] Aziz I., Islam S.U., Fayyaz M. and Azram M. (2014): New algorithms for numerical assessment of nonlinear integrodifferential equations of second-order using Haar wavelets.- Walailak Journal of Science and Technology, vol.12, pp.995-1007.

[23] Saeed U. and Rehman M.U. (2013): Haar wavelet-quasilinearization technique for fractional nonlinear differential equations.- Applied Mathematics and Computation, vol.220, pp.630-648.

[24] Kaur H., Mittal R.C. and Mishra V. (2011): Haar wavelet quasilinearization approach for solving nonlinear boundary value problems.- American Journal of Computational Mathematics, vol.1, pp.176-182. 
[25] Jiwari R. (2012): A Haar wavelet quasilinearization approach for numerical simulation of Burgers' equation.Computer Physics Communications, vol.183, pp.2413-2423.

[26] Lepik Ü. (2005): Numerical solution of differential equations using Haar wavelets.- Mathematics and Computers in Simulation, vol.68, pp.127-143.

[27] Afzal N. (2003): Momentum transfer on power law stretching plate with free stream pressure gradient.-International Journal of Engineering Science, vol.41, pp.1197-1207.

[28] Bellman R.E. and Kalaba R.E. (1965): Quasilinearization and Nonlinear Boundary-Value Problems.- American Elsevier Publishing Company, New York.

[29] Majak J., Shvartsman B.S., Kirs M., Pohlak M. and Herranen H. (2015): Convergence theorem for the Haar wavelet based discretization method.- Composite Structures, vol.126, pp.227-232.

[30] Sachdev P.L., Bujurke N.M. and Awati V.B. (2005): Boundary value problems for third-order nonlinear ordinary differential equations.- Studies in Applied Mathematics, vol.115, pp.303-318.

[31] Kudenatti R.B., Awati V.B. and Bujurke N.M. (2011): Approximate analytical solutions of a class of boundary layer equations over nonlinear stretching surface.- Applied Mathematics and Computation, vol.218, pp.2952-2959.

[32] Sakiadis B.C. (1961): Boundary-layer behavior on continuous solid surfaces: II. The boundary layer on a continuous flat surface.- AIChE Journal, vol.7, pp.221-225.

[33] Hayat T., Zaman H. and Ayub M. (2011): Analytical solution of hydromagnetic flow with Hall effect over a surface stretching with a power-law velocity.- Numerical Methods for Partial Differential Equations, vol.27, pp.937-959.

Received: January 14, 2021

Revised: March 25, 2021 